\title{
Modeling of cytochrome P-450 enzyme inhibitors activities using 2D/3D QSAR
}

\author{
Farahmand Asadi ${ }^{1} \mathbb{D} \cdot$ Mohammad Hossein Fatemi $^{1}$
}

Received: 13 April 2020 / Accepted: 10 August 2020 / Published online: 28 August 2020

(c) Springer Nature Switzerland AG 2020

\begin{abstract}
In this research, quantitative structure-activity relationship (QSAR) studies were carried out on the inhibitory activities of a set of nicotine derivatives against the cytochrome-p450 2A6 (CYP2A6) enzyme. Two-dimensional quantitative structure-activity relationship (2D-QSAR) models were developed using multiple linear regression (MLR) and linear square-support vector machine (LS-SVM) methods. The result of statistical parameters of the MLR method show that the correlation coefficient $\left(R^{2}\right)$ and standard error $(S E)$ for the training set respectively are $R^{2}=0.702, S E=0.49$ and for the test set $R^{2}=0.689, S E=0.52$ and the results of statistical parameters of the $L S-S V M$ method for the training set $R^{2}=0.993$, $\mathrm{SE}=0.10$ and for the test set $\mathrm{R}^{2}=0.977, \mathrm{SE}=0.20$. The obtained results reveal the superiority of LS-SVM over MLR model. Then three-dimensional quantitative structure-activity relationship (3D-QSAR) model was developed using comparative molecular field analysis (CoMFA) and comparative molecular similarity indices analysis (CoMSIA) on the same dataset of nicotine derivatives. The acquired statistical parameters of the CoMFA model for the training set are $\mathrm{R}^{2}=0.884, \mathrm{SE}=0.316$ and for the test set $R^{2}=0.847, \mathrm{SE}=0.33, \mathrm{~F}=41.27, \mathrm{Q}^{2}=0.581$, while the statistical values of CoMSIA model for the training set are $\mathrm{R}^{2}=0.889, \mathrm{SE}=0.31$ and for the test set $\mathrm{R}^{2}=0.670, \mathrm{SE}=0.54, \mathrm{~F}=39.049, \mathrm{Q}^{2}=0.554$. The results of this study revealed that the CoMFA model was more predictive and could be helpful in designing novel potent nicotine derivatives with enhanced inhibitory activity.
\end{abstract}

Keywords CoMFA · CoMSIA · Cytochrome P-450 2A6 · Inhibitory activity · LS-SVM · MLR

\section{Introduction}

Tobacco contains a package of harmful and addictive compounds [2] that its all forms rise the health problems in humans [47]. Long-term is speaking of lung cancer, heart disease, stroke and other fatal and non-fatal diseases [7, $23,33]$. Tobacco is the common cause of premature death compared to other consumer products [37]. It kills more people in developing countries than in industrialized countries [18]. The principal ingredient of tobacco leaves is nicotine which is highly addictive [3]. Even though numerous smoking-related diseases are preventable but due to the dependency of a smoker to nicotine, only a small number of smokers manage to quit perfectly. Researchers have developed different nicotine replacements like nicotine gum and patch, to lower the addiction liability and to assist in smoking cessation [44]. The metabolism of nicotine leads to several metabolites [26]. In humans, the major product of this conversion is cotinine [4]. Hepatic Cytochrome P-450 2A6 (CYP2A6) is widely considered to be the principal catalyzing enzymes of nicotine metabolism via the intermediacy of aldehydes-catalyzed iminium ion $[24,36]$. The addiction liability of nicotine is closely related to the efficiency of the CYP2A6- medicated metabolism of nicotine and a specific concentration of nicotine in the bloodstream. Individuals with inactive CYP2A6

Farahmand Asadi, farahmand361@gmail.com | ${ }^{1}$ Chemometrics Laboratory, Faculty of Chemistry, University of Mazandaran, Babolsar 47416-97445, Iran. 
alleles displayed a significantly decreased dependency on smoking $[34,38]$. Since nicotine primarily oxidation by the CYP2A6 is a rate-limiting step, obstructing this enzymatic reaction by a chemical inhibitor would increase nicotine oral bioavailability and decrease smoking [40]. Until today, a variety of compounds have been tested as CYP2A6 inhibitors with strong inhibitory results. To be used as a CYP2A6 inhibitory ligand, the best possible candidate of the drug must be an extremely potent and selective agent to avoid undesirable side effects. In this study, a series of nicotine derivatives with noun inhibitory properties were used as potent inhibitors of CYP2A6 enzyme [15]. The reported compounds were used as the database for performing two-dimensional quantitative structure-activity relationship (2D-QSAR) and three-dimensional quantitative structure-activity relationship (3D-QSAR) studies. Denton and co-workers was mentioned the inhibitory activity (IC50) of a large number of nicotine analogue that highly selective for the inhibition of CYP2A6 versus the other isoforms of CYP contains CYP2E1, CYP2B6, CYP2C9, CYP2C19 and CYP2D6; Also, there is no effects of nicotine on activity of human CYP2E1 \{Hukkanen, 2010 \#458\} and CYP1A2 \{Hukkanen, 2011 \#457\}. 2D-QSAR study applied in a search for aminooxadiazole derivatives as PIM1 inhibitors \{Aouidate, $2018 \# 521\}$, N-substituted oseltamivir derivatives as potent avian influenza virus H5N1 inhibitors \{Chtita, 2020 \#524\} and investigation of a class of acetylcholinesterase inhibitors being pyridazine derivatives as Anti-Alzheimer drugs \{Zerroug, 2019 \#525\}. Also, 3D-QSAR applied for designing of new Kinase-related apoptosis-inducing kinase 2 (DRAK2) inhibitors \{Aouidate, 2018 \#520\} and investigation of Furanone derivatives as new inhibitors of Cell division cycle 7 (CDC7) kinase \{Aouidate, 2018 \#522\} and pyrazolopyrimidine derivatives as $\mathrm{Ca}^{2+} /$ calmodulin-dependent protein kinase II (CAMKII $\delta$ ) kinase inhibitors \{Aouidate, $2018 \# 523\}$. 2D-QSAR models were developed using multiple linear regression (MLR) and linear square-support vector machine (LS-SVM) methods. The purpose of this study was to extract a good correlation between the biological activity of nicotine derivatives and their 3D structure with the eventual aim of predicting and developing a potent and specific inhibitor of CYP2A6 enzyme. 3D-QSAR models consisting of comparative molecular field analysis (COMFA) [32] and comparative molecular similarity indices analysis (COMSIA) [5, 31] were used to forecast activities of these inhibitors successfully and to provide areas in space where various interactive fields may affect the activity. The predictive inhibitory activity values could be anticipated by each generated 3D-QSAR models. Moreover, the subsequent partial least squares (PLS) analysis of the data was used to construct a linear correlation coefficient between experimental versus calculated inhibitory activities [49]. Finally, COMFA and COMSIA contour maps are used to describe different structural necessities for the inhibitors in the active site of CYP2A6. Contour results can be helpful in testing the new designed analogues before their synthesis.

\section{2D-QSAR study}

\subsection{Dataset and molecular modeling for 2D-QSAR}

A substituted pyridine analogues of nicotine with wellexpressed inhibitory activity $\left(I_{50}\right)$ against the CYP2A6 enzyme were used for the current study [15]. The IC50 value is the minimum concentration of a compound that results in a 50\% reduction in the activity of CYP2A6 enzyme that were transformed into the corresponding form of the logarithm $\left(\mathrm{plC}_{50}\right)$. The molecular structure of the studied compounds with the corresponding $\mathrm{pIC}_{50}$ is shown in Table 1 . Based on the measured values, compounds numbered 6,18 and 60 possess inhibitory activity values greater than $67 \mu \mathrm{m}$, so these compounds were excluded from the current QSAR modeling. All the remaining compounds then randomly divided into two independent subsets; a training set (49 compound) for the generation of QSAR models and a test set $(8 \mathrm{com}$ pound) for evaluating the quality of the models. The training set was selected manually based on the diversity of structures possessing a wide range of activity. As shown in this table, compounds marked with $\left({ }^{*}\right)$ symbol represents the test set and the rest are the training set. The selection of the test set was in such a way that their values lie between the maximum-minimum values of the training set.

\subsection{Principal component analysis (PCA)}

The principal component analysis (PCA) [48] is a powerful way to reduce the dimensions of a set of multivariate data. The number of employed descriptors represents the dimensions of the data set which are often related to each other. This analysis is used to depict multivariate data through diversity plots and reduce the number of variables in a series of non-related variables which is most commonly used for sample analysis in the applicability domain. Using molecular descriptors, primary PCA was carried out on the remaining 57 compounds with diverse molecular structures. As can be seen in Fig. 1, the results of PCA show well- distribution of chemicals in the structural space except for compounds numbered 36,38 and 39 which were then excluded from the consequent 2D-QSAR modeling and the remaining compounds were used in the current study. 
Table 1 Structure and experimental inhibitory activities of nicotine analogues on CYP2A6 enzyme

\begin{tabular}{|c|c|c|c|}
\hline Compound & Structure & $\mathrm{IC}_{50}(\mu \mathrm{M})$ & $\mathrm{pIC}_{50}(\mu \mathrm{M})$ \\
\hline 1 & & 4.40 & -0.64 \\
\hline 2 & & 1.20 & -0.08 \\
\hline 3 & & 3.20 & -0.51 \\
\hline 4 & & 0.10 & 1.00 \\
\hline 5 & & 0.21 & 0.68 \\
\hline 6 & & $\geq 67$ & -1.83 \\
\hline & & 1.50 & -0.18 \\
\hline 8 & & 0.79 & 0.10 \\
\hline 9 & & 1.40 & -0.15 \\
\hline 10 & & 19.70 & -1.29 \\
\hline 11 & & 0.22 & 0.66 \\
\hline 12 & & 0.97 & 0.01 \\
\hline 13 & & 9.70 & -0.99 \\
\hline 14 & & 4.50 & -0.65 \\
\hline 15 & & 0.25 & 0.60 \\
\hline 16 & & 2.60 & -0.41 \\
\hline 17 & & 6.60 & -0.82 \\
\hline 18 & & $\geq 67$ & -1.83 \\
\hline 19 & & 7.70 & -0.89 \\
\hline 20 & & 43.70 & -1.64 \\
\hline 21 & & 5.10 & -0.71 \\
\hline 22 & & 2.70 & -0.43 \\
\hline
\end{tabular}

\subsection{Descriptors calculation and selection}

Initially, the structure of the compounds was drawn using hyperchem software [27]. Then, its three-dimensional structure was optimized using the AM1 semi-empirical method. The resulting output files functioned as input for Dragon [35], MOPAC [42], CODESSA [28] and PADEL [50] software to calculate the molecular descriptors. But only a few of them have an effective correlation with the inhibitory activity of CYP2A6 enzyme. Besides, there is a 
Table 1 (continued)

\begin{tabular}{|c|c|c|c|}
\hline Compound & Ctructure & $\mathrm{IC}_{50}(\mu \mathrm{M})$ & $\mathrm{pIC}_{50}(\mu \mathrm{M})$ \\
\hline 23 & & 9.80 & -0.99 \\
\hline 24 & & 1.80 & -0.26 \\
\hline 25 & & 27.40 & -1.44 \\
\hline 26 & & 1.10 & -0.04 \\
\hline 27 & & 6.30 & -0.80 \\
\hline 28 & & 44.50 & -1.65 \\
\hline 29 & & 0.25 & 0.60 \\
\hline 30 & & 6.20 & -0.79 \\
\hline 31 & & 0.24 & 0.62 \\
\hline 32 & & 0.24 & 0.62 \\
\hline 33 & & 0.71 & 0.15 \\
\hline 34 & & 13.70 & -1.14 \\
\hline 35 & & 0.02 & 1.70 \\
\hline 36 & & 1.20 & -0.08 \\
\hline 37 & & 0.04 & 1.40 \\
\hline 38 & & 1.50 & -0.08 \\
\hline 39 & & 2.80 & -0.45 \\
\hline 40 & & 0.18 & 0.74 \\
\hline 41 & & 0.28 & 0.55 \\
\hline 42 & & 22.20 & -1.35 \\
\hline 43 & & 47.20 & -1.67 \\
\hline
\end{tabular}

high intercorrelation between many descriptors. Thus, descriptors, having zero, constant or almost constant values were removed from the pool of descriptors because they could not link the differences in molecule structure to the differences in activity. The correlation coefficient between the remaining descriptors was calculated and those having $r^{2}>0.9$ were considered as intercorrelated because contain the same information or effect on activity and utilization of one of them is sufficient for modeling. Afterward, forward stepwise variable selection 
Table 1 (continued)

\begin{tabular}{|c|c|c|c|}
\hline Compound & Structure & $\mathrm{IC}_{50}(\mu \mathrm{M})$ & $\mathrm{pIC}_{50}(\mu \mathrm{M})$ \\
\hline 44 & & 5.60 & -0.75 \\
\hline 45 & & 35.20 & -1.55 \\
\hline 46 & & 0.09 & 0.05 \\
\hline 47 & & 0.89 & 0.05 \\
\hline 48 & & 22.70 & -1.36 \\
\hline 49 & & 1.40 & -0.15 \\
\hline 50 & & 11.00 & -1.04 \\
\hline 51 & & 0.17 & 0.77 \\
\hline 52 & & 0.59 & 0.23 \\
\hline 53 & & 0.13 & 0.89 \\
\hline 54 & & 0.52 & 0.28 \\
\hline 55 & & 0.23 & 0.64 \\
\hline 56 & & 0.17 & 0.77 \\
\hline 57 & & 0.25 & 0.60 \\
\hline 58 & & 0.62 & 0.21 \\
\hline 59 & & 64.80 & -1.81 \\
\hline 60 & & $\geq 67$ & -1.83 \\
\hline
\end{tabular}

was used to further reduce non-significant descriptors. The selected descriptors served as inputs for establishing multiple linear regressions (MLR) and least- squares support vector machine (LS-SVM) models. The correlation coefficient matrix between the selected descriptors is depicted in the Table 2. As shown in this table, the selected descriptors are completely independent of each other.

\subsection{Multiple linear regression}

The most common method utilized in QSAR modeling is multiple linear regression $[1,14]$. Indeed, MLR is referred to be the classic and standard technique for analysis of multivariate data. The main aim of multiple regression is to quantify a linear relationship between a dependent variable (inhibitory activity) and a number of independent 


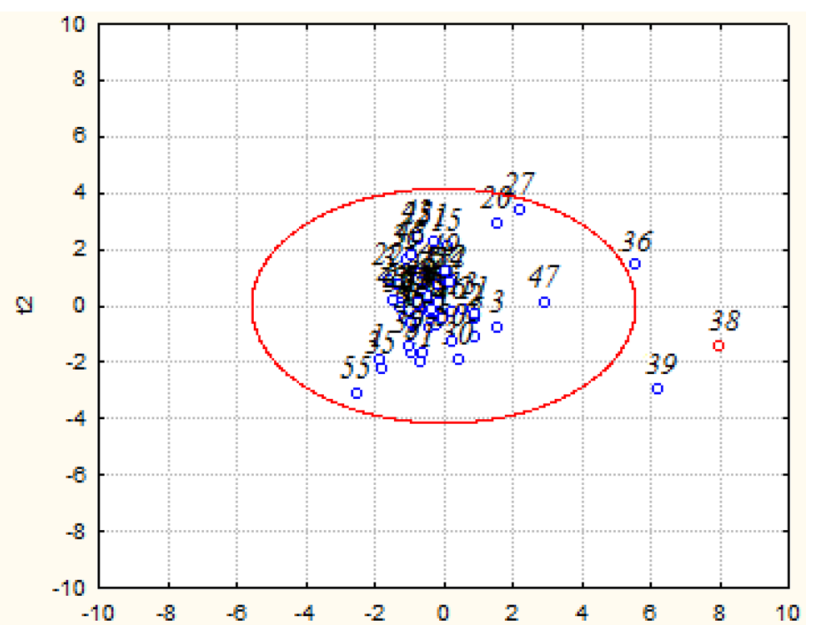

Fig. 1 The plot of accomplished PCA analysis

variables (descriptors). This method of regression utilizes the least square curve fitting strategy to determine the values of regression coefficients. The multivariate analysis applies statistical methods to analyze metrics of multidimensional data such as dependent and/or independent variables. The multiple linear regression equation is shown below;

$\hat{y}=b_{0}+b_{1} x_{1}+b_{2} x_{2}+b_{p} x_{p}$

where $\hat{y}$ is the dependent variable and $b_{1}$ and $b_{2}$ represent the regression coefficients and $b_{0}$ is a regression constant and $x_{1}$ to $x_{p}$ are independent variables.

\subsection{Least-squares support vector machine}

Support vector machine (SVM), developed by Curtis and Vapnik [11] obtained popularity in a variety of academic works due to its many attractive characteristics and remarkable empirical performance. Later an alternative version of SVM through the implementation of the leastsquares supplement, was developed by Suykens and Vandewalle [43]. The main advantage of LS-SVM is its capacity for dealing with problems of linear and nonlinear multivariate capacity in a rather fast way. Further comprehensive studies of LS-SVM approach could be found in references $[9,13,19]$. On the whole, a linear relationship between the dependent variable $(y)$ and the regressors $(x)$ are always fitted by LS-SVM as following:

$\mathrm{y}=\sum_{i=1}^{N} \alpha_{i} \mathrm{k}\left(\mathrm{x}_{\mathrm{i}}, \mathrm{x}\right)+\mathrm{b}$

$\alpha_{\mathrm{i}}=2 \gamma \mathrm{e}_{\mathrm{i}}$

where $a_{i}$ represents Lagrange multipliers, $k\left(x_{i}, x\right)$ is the kernel function, $b$ represents the bias term and $\gamma$ (gamma) parameter denotes the relative weight of the error term. A polynomial or Gaussian form of kernel function is utilized to delineate the input vectors into a higher dimensional feature space. The radial basis function (RBF) is considered the most common kernel function:

$\mathrm{k}\left(\mathrm{x}_{\mathrm{i}}, \mathrm{x}\right)=\exp \left(-\left\|\mathrm{x}_{\mathrm{i}}-\mathrm{x}_{\mathrm{j}}\right\|^{2} / 2 \sigma\right)$

where $\sigma^{2}$ represents the width of the RBF function. Generalizability potential of SVM relies on the appropriate choice of its parameters. The most careful attention in the LS-SVM algorithm concerns the choice of kernel function and its respective parameters. Since they clarify the spread of training set samples in the high dimensional feature space. Subsequent to training the predictability power of the machine is utilized to foresee or estimate unknown target values of objects. In this study, LS-SVM was established by RBF kernel function and MATLAB (v.7.6) was used to carry out all the calculations related to this work.
Table 2 Correlation matrix among selected descriptors

\begin{tabular}{|c|c|c|c|c|c|c|c|c|}
\hline & $\mathrm{Hy}$ & Mor21m & MATS8m & Mor18u & BEHm2 & $\mathrm{A} \log \mathrm{P}$ & $\mathrm{L} 2 \mathrm{u}$ & Mor20u \\
\hline $\mathrm{Hy}$ & 1 & -0.072 & 0.344 & 0.024 & -0.146 & 0.106 & 0.201 & 0.202 \\
\hline Mor21m & & 1 & -0.407 & 0.300 & 0.132 & -0.177 & 0.416 & 0.295 \\
\hline MATS8m & & & 1 & -0.233 & 0.345 & 0.152 & 0.222 & 0.388 \\
\hline Mor18u & & & & 1 & -0.231 & 0.461 & 0.122 & -0.150 \\
\hline BEHm2 & & & & & 1 & 0.062 & 0.162 & -0.120 \\
\hline$A \log P$ & & & & & & 1 & -0.046 & 0.001 \\
\hline $\mathrm{L} 2 \mathrm{u}$ & & & & & & & 1 & 0.198 \\
\hline Mor20u & & & & & & & & 1 \\
\hline
\end{tabular}




\section{3D-QSAR study}

\subsection{Datasets}

The same selected training and test set in 2D-QSAR modeling was applied for developing the 3D-QSAR models.

\subsection{Molecular modeling}

The three-dimensional structures of 57 nicotine derivatives were built by using Sybyl (x.2.1.1) programming package of Tripos, Inc [6]. The whole compounds were subjected to structural energy minimization by using Sybyl energy minimizer (Tripos Force Field) and the atomic charge was assigned by Gasteiger-Huckel method, with Powell conjugate gradient algorithm with $0.005 \mathrm{kcal} \mathrm{mol}^{-1}$ $\AA^{-1}$ convergence criterion.

\subsection{Molecular alignment}

The most sensitive parameter in CoMFA and CoMSIA studies is the alignment of 3D structures of the compounds to be analyzed in accord with an appropriate conformational template. The prediction power and accuracy of CoMFA and CoMSIA models and the creditability of contour maps directly rely on the suitable alignment molecule. In this study, the most active compound 35 was employed as a template for alignment and other compounds were superimposed on it, the resulting alignments are depicted in Fig. 2. As can be seen from this figure, the common fragment for all compounds is a pyridine ring with 3-substituted heteroaromatics.

\subsection{CoMFA and CoMSIA analyses}

In CoMFA method [32] the biological activity of a series of compounds are related to their steric and electrostatic

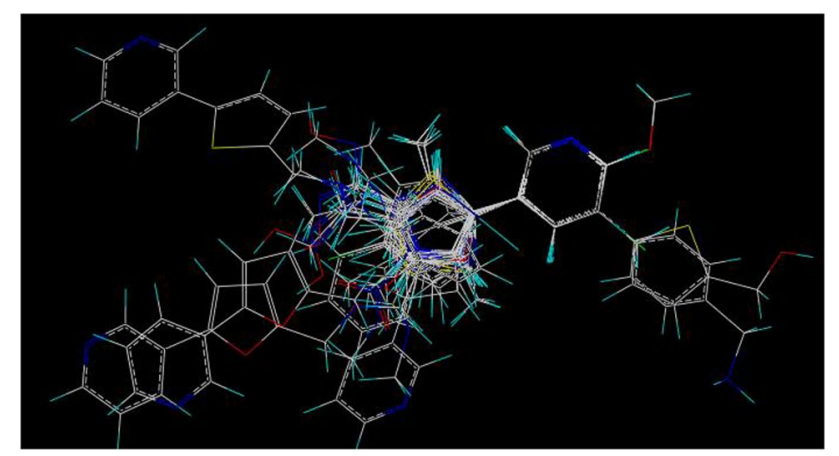

Fig. 2 Structural alignment of the training set molecules for establishing the 3-D QSAR models fields which are derived by putting the aligned compounds one by one into a 3D grid box with regularly grid spacing of $2 \AA$ and expanding beyond $4 \AA$ in all directions from the coordination of each compound [30]. The Tripos force field approach is then used to calculate the van der walls potentials and Coulombic terms which respectively stand for steric and electrostatic fields. A sp ${ }^{3}$ carbon atom having a +1 charge and a van der walls radius of $1.52 \AA$ was employed as a probe atom to compute the electrostatic and steric fields at each grid intersection. The threshold values of column-filtering was adjusted to $2.0 \mathrm{kcal}$. $\mathrm{mol}^{-1}$ to enhance signal-noise ratio by deleting those grid points having a energy values lower than this threshold. It is needed to truncate the energy values of steric and electrostatic fields to $30 \mathrm{kcal} \mathrm{mol}^{-1}$ to prevent the energy values within the molecules from stretching into infinity $[10,12]$.

In CoMSIA [31] method which is an extension of the CoMFA method, five descriptors including steric, electrostatic, hydrophobic, hydrogen bond donor and hydrogen bond acceptor were computed with the same grid box and probe atom utilized in CoMFA calculations [5]. Gaussian type functional distance dependence was applied in CoMSIA which can avoid a sharp change of potential energies adjacent to the molecular surface using a default value of 0.3 as the attenuation Factor [40]. The equation for the calculation of similarity indices $\left(A_{F}\right)$ between the molecules and the probe atom can be shown as below:

$A_{F, K}^{q}(j)=\sum_{i} \omega_{\text {probe, },} \omega_{i k} e_{i q}^{-\alpha r 2}$

where $\omega_{\text {probe, }}$ represents probe atom, $\omega_{\mathrm{ik}}$ represents the real value of the physiochemical property $k$ of atom $i, a$ denotes the attenuation factor and $r_{\text {iq }}$ represents the mutual distance between each atoms $i$ of the molecule $j$ and the probe atom at lattice point $\mathrm{q}$.

\subsection{Partial least square analysis and model validation}

After the calculation of all CoMFA and CoMSIA descriptors, partial least squares (PLS) analysis, supposed to be an extension of the multiple regression analysis, was carried out to produce a 3D-QSAR model $[8,41]$. The PLS regression algorithm was employed to construct a linear relationship between inhibitory activity values $\left(\mathrm{pIC}_{50}\right)$ as dependent variables and CoMFA and CoMSIA fields as independent variables. Before performing the PLS analysis, column filtering was employed to filter CoMFA and CoMSIA columns to a limit smaller than $20 \mathrm{kcal} \mathrm{mol}^{-1}$ to lower the noise. In order to achieve the excellent model, the cross- validation process was done using the 
leave-one-out (LOO) approach to investigate the predictability of the generated model and to determine the optimal number of components (ONC) giving rise to the utmost cross-validated $r^{2}\left(r_{c v}^{2}\right)$ [22]. The LOO methodology is based on omitting one molecule from the dataset and then, driving a model using the remaining molecules. Afterwards, the generated model is employed to predict the activity of the deleted molecule. The ONC achieved from cross-validation techniques was subsequently employed in the regression model. The PLS analysis based on non-cross validation was then utilized to create final models of CoMFA and CoMSIA. To further appraise the robustness and statistical reliance of the accurate model, a bootstrap analysis for 100 cycles was done. In this technique, $n$ chance selections among $n$ objects of the original set are executed several times to achieve an excellent statistical result (100 times are needed). In each selection,

\section{Results and discussion}

\subsection{D-QSAR statistical analyses}

The technique of the stepwise multi linear regression was used to choose the most related descriptors from the pool of the descriptors which rely on the compounds of the training set. Based on the calculation outcomes of the correlation matrix in Fig. 2 no intense correlation was found among the chosen descriptors. Moreover, the MLR approach was employed to construct linear models of QSAR to foresee the inhibitory activities of nicotine derivatives. The selected descriptors were employed as independent variables and $\mathrm{pIC}_{50}$ values were employed as dependent variables. Specification of the achieved MLR model with these descriptors is depicted in the following equation:

$$
\begin{aligned}
\mathrm{PIC}_{50}= & 4.892( \pm 2.426)+0.998( \pm 0.209) \times \mathrm{Hy}+2.738( \pm 0.793) \times \text { MATs } 8 \mathrm{~m} \\
& -0.502( \pm 0.265) \times \mathrm{L} 2 \mathrm{u}+0.446( \pm 0.149) \times \text { AlogP }-1.601( \pm 0.705) \times \mathrm{BEHm} 2 \\
& -0.763( \pm 0.450) \times \text { Mor } 20 \mathrm{u}+2.738( \pm 0.793) \times \text { Mor } 21 \mathrm{~m}-1.984( \pm 0.398) \times \text { Mor } 18 \mathrm{u} \\
\mathrm{n}= & 46 \mathrm{R}^{2}=0.702 \mathrm{SE}=.49 \mathrm{~F}=10.896
\end{aligned}
$$

some objects may be excluded from the PLS analysis, while some objects may be incorporated more than one time. Bootstrap $r^{2}\left(r_{\text {boot }}^{2}\right)$ stands for the mean correlation coefficient. To validate the obtained models of CoMFA and CoMSIA, the predictabity power for the compounds of the test set (represented as $r_{\text {pred }}^{2}$ ) is verified by employing the following formula:

$r_{\text {pred }}^{2}=(S D-P R E S S) / S D$

where SD represents the sum of the squared deviations found between the activities of the external test set and training set molecules and PRESS represents the sum of the squared deviations found between the actual and predicted activities of the external test set molecules. where throughout this study, $\mathrm{n}$ stands for the number of compounds, $R^{2}$ depicts the square of correlation coefficients, SE represents the estimated standard error and $\mathrm{F}$ represents the F-ratio between the variances of computed and observed activities. Also, the values shown in the parenthesis depict the standard errors related to the regression coefficient. Definition and categories of the 8 selected descriptors were appeared in Table 3. Further detailed studies of these descriptors can be acquired in the handbook of molecular descriptors [45].

The Hy stands for hydrophilic index descriptor and is related to the hydrophilicity of the compound. It is calculated as below:
Table 3 Definition and categories of selected descriptors

\begin{tabular}{lll}
\hline Descriptor & Definition & Category \\
\hline Hy & Hydrophilicity factor & Molecular properties \\
Mor18u & 3-D MoRSE signal - 18/unweighted & 3-D MoRSE \\
Mor20u & 3-D MoRSE signal - 20/unweighted & 3-D MoRSE \\
Mor21m & 3-D MoRSE signal - 21/weighted by atomic mass & 3-D MoRSE \\
MATS8m & Moran autocorrelation - lag 8/weighted by atomic mass & 2-D autocorrelation \\
BEHm2 & Highest eigen value no.2 of Burden matrix/weighted by atomic mass & BCUT descriptors \\
AlogP & Ghose-Crippen octanol-water partition coefficients. (logP) & Molecular properties \\
L2u & 2nd component size directional WHIM index/unweight & WHIM descriptors \\
\hline
\end{tabular}


$\mathrm{Hy}=\frac{\left(1+N_{H y}\right) \cdot \log _{2}\left(1+N_{H y}\right)+N_{c} \cdot\left(\frac{1}{A} \cdot \log _{2}^{\frac{1}{A}}\right)+\sqrt{\frac{N_{H y}}{A^{2}}}}{\log _{2}^{(1+A)}}$

where $\mathrm{N}_{\mathrm{Hy}}$ depicts the number of hydrophilic sets $(-\mathrm{OH}$, $-\mathrm{SH},-\mathrm{NH}), \mathrm{N}_{\mathrm{c}}$ defines the number of carbon atoms and A depicts the number of atoms disregarding hydrogen atoms [44]. The negative coefficient of $\mathrm{H}_{\mathrm{y}}$ descriptor signifies that presence of hydrophilic groups in aromatic rings decreases $\mathrm{plC}_{50}$. It is important to mention that a low value of $\mathrm{plC}_{50}$ shows a low inhibitory activity of inhibitors. Mor18u and Mor20u are 3D-MoRSE (3D- molecular representation of structure based on electron diffraction) un-weighted descriptors and encode information of structural features like mass and number of branching in substituted benzene derivative [17]. The negative sign in the regression coefficients of these descriptors reveals that the addition of substituents in the aromatic ring decrease the value of pIC50. The fourth descriptor is Mor21 m, 3D-MoRSE - signal 21/weighted by atomic masses $[20,39]$. The positive sign in the regression coefficient of this descriptor reflects positively the role of atomic masses on the inhibitory activity of inhibitors. The fifth descriptor is MATs $8 \mathrm{~m}$, Moran autocorrelation- lag8 which is weighted by atomic mass and is 2D- autocorrelation [16]. The positive sign in the regression coefficient of this descriptor denotes positively increased value of $\mathrm{pIC}_{50}$. The sixth descriptor is BEHm2, highest eigen value NO. 2 of Burden matrix, which is weighted by atomic mass [29]. Encoding of this descriptor is accompanied by descriptor of Burden eigen values and is computed using hydrogen involved molecular graph. As shown in Eq. (7), the regression coefficient of this descriptor has a negative sign which indicates a negative effect of adding hydrogen in molecular graph on the $\mathrm{plC}_{50}$ value. The next descriptor is AlogP, Ghose-Crippen octanol-water partition coefficient, which denotes the lipophilicity of the molecule [21]. The positive coefficient of this descriptor in Eq. (7) shows that as the hydrophobic groups of the molecule increase the inhibitory activity becomes stronger. The last descriptor is $L 2 u$, 2nd component size directional WHIM index/unweight, which uses molecular size to gain related molecular 3D information [46]. According to Eq. (7), this descriptor has a negative coefficient which means it is inversely related to the $\mathrm{pIC}_{50}$. These selected descriptors were employed as inputs to drive linear and non-linear 2D-QSAR models respectively by MLR and LS-SVM methods.

In LS-SVM modeling, the values of parameters of $\sigma^{2}$ and the relative weight of the regression error $(\gamma)$ were optimized to gain the maximum root mean square errors (RMSE). Consequently, the optimum values of $\sigma^{2}$ and $\gamma$ were found 8 and 98 , respectively. The experimental and
Table 4 Dataset and corresponding experimental and calculated $\mathrm{plC}_{50}$ values for MLR and LS-SVM models

\begin{tabular}{|c|c|c|c|c|c|}
\hline \multirow[t]{2}{*}{ Compound } & \multirow[t]{2}{*}{$\mathrm{plC}_{50 \exp }$} & \multicolumn{2}{|l|}{ MLR } & \multicolumn{2}{|l|}{ LS-SVM } \\
\hline & & $\mathrm{plC}_{50 \text { PRED }}$ & Residual & $\mathrm{plC}_{50 \text { PRED }}$ & Residual \\
\hline 1 & -0.64 & -0.57 & -0.07 & -0.61 & -0.03 \\
\hline 2 & -0.08 & 0.01 & -0.09 & -0.03 & -0.05 \\
\hline 3 & -0.51 & 0.28 & -0.79 & -0.47 & -0.04 \\
\hline 4* & 1.00 & 0.32 & 0.68 & 0.51 & 0.49 \\
\hline 5 & 0.68 & 0.37 & 0.31 & 0.68 & 0.00 \\
\hline 7 & -0.18 & -0.13 & -0.05 & -0.18 & 0.00 \\
\hline 8 & 0.10 & 0.39 & -0.29 & 0.08 & 0.02 \\
\hline 9 & -0.15 & -0.74 & 0.59 & -0.19 & 0.04 \\
\hline 10 & -1.29 & -0.87 & -0.42 & -1.35 & 0.06 \\
\hline 11 & 0.66 & 0.41 & 0.25 & 0.63 & 0.03 \\
\hline $12^{*}$ & 0.01 & -0.21 & 0.22 & -0.15 & 0.16 \\
\hline 13 & -0.99 & -0.59 & -0.40 & -0.98 & -0.01 \\
\hline 14 & -0.65 & -0.71 & 0.06 & -0.64 & -0.01 \\
\hline $15^{*}$ & 0.60 & -0.05 & 0.65 & 0.06 & 0.54 \\
\hline 16 & -0.41 & -0.39 & -0.02 & -0.40 & -0.01 \\
\hline 17 & -0.82 & -0.89 & 0.07 & -0.82 & 0.00 \\
\hline $19^{*}$ & -0.89 & -0.61 & -0.28 & -0.90 & 0.01 \\
\hline 20 & -1.64 & -1.33 & -0.31 & -1.59 & -0.05 \\
\hline 21 & -0.71 & -0.91 & 0.20 & -0.69 & -0.02 \\
\hline 22 & -0.43 & -0.32 & -0.11 & -0.29 & -0.14 \\
\hline 23 & -0.99 & -0.46 & -0.53 & -0.99 & 0.00 \\
\hline $24^{*}$ & -0.26 & -0.28 & 0.02 & -0.77 & 0.51 \\
\hline 25 & -1.44 & -0.63 & -0.81 & -1.11 & -0.33 \\
\hline 26 & -0.04 & -0.75 & 0.71 & -0.43 & 0.39 \\
\hline 27 & -0.80 & -1.07 & 0.27 & -0.74 & -0.06 \\
\hline 28 & -1.65 & -1.40 & -0.25 & -1.65 & 0.00 \\
\hline 29 & 0.60 & 0.49 & 0.11 & 0.48 & 0.12 \\
\hline 30 & -0.79 & 0.06 & -0.85 & -0.52 & -0.27 \\
\hline 31 & 0.62 & -0.22 & 0.84 & 0.40 & 0.22 \\
\hline 32 & 0.62 & 0.16 & 0.46 & 0.66 & -0.04 \\
\hline 33 & 0.15 & -0.35 & 0.50 & 0.11 & 0.04 \\
\hline 34 & -1.14 & -0.24 & -0.90 & -1.14 & 0.00 \\
\hline 35 & 1.70 & 0.92 & 0.78 & 1.54 & 0.16 \\
\hline 37 & 1.40 & -1.02 & 0.38 & 1.36 & 0.04 \\
\hline 40 & 0.74 & 0.15 & 0.59 & 0.74 & 0.00 \\
\hline 41 & 0.55 & -0.31 & 86,0 & 0.48 & 0.07 \\
\hline 42 & -1.35 & -1.32 & -0.03 & -1.33 & -0.02 \\
\hline 43 & -1.67 & -1.99 & 0.32 & -1.67 & 0.00 \\
\hline 44 & -0.75 & 0.01 & -0.76 & -0.63 & -0.12 \\
\hline $45^{*}$ & -1.55 & -0.40 & -1.15 & -1.52 & -0.03 \\
\hline 46 & 1.05 & 1.00 & 0.05 & 1.03 & 0.02 \\
\hline 47 & 0.05 & 0.02 & 0.03 & 0.05 & 0.00 \\
\hline 48 & -1.36 & -1.67 & 0.31 & -1.35 & -0.01 \\
\hline 49 & -0.15 & 0.53 & -0.68 & -0.14 & -0.01 \\
\hline 50 & -1.04 & -0.51 & -0.53 & -1.05 & 0.01 \\
\hline 51 & 0.77 & 0.67 & 0.10 & 0.77 & 0.00 \\
\hline 52 & 0.23 & 1.00 & -0.77 & 0.37 & -0.14 \\
\hline $53^{*}$ & 0.89 & 0.66 & 0.23 & 0.60 & 0.29 \\
\hline
\end{tabular}


Table 4 (continued)

\begin{tabular}{lrrrrrr}
\hline Compound & $\mathrm{pIC}_{50 \text { exp }}$ & \multicolumn{2}{l}{$\mathrm{MLR}$} & & \multicolumn{2}{l}{ LS-SVM } \\
\cline { 3 - 4 } \cline { 6 - 7 } \cline { 6 - 7 } & & $\mathrm{plC}_{50 \text { PRED }}$ & Residual & & plC $C_{50 \text { PRED }}$ & Residual \\
\hline $\mathbf{5 4}$ & 0.28 & 0.58 & -0.30 & & 0.30 & -0.02 \\
$\mathbf{5 5}$ & 0.64 & 0.18 & 0.46 & & 0.62 & 0.02 \\
$\mathbf{5 6}$ & 0.77 & 0.25 & 0.52 & & 0.62 & 0.15 \\
$\mathbf{5 7}$ & 0.60 & 0.28 & 0.32 & & 0.58 & 0.02 \\
$\mathbf{5 8 *}$ & 0.21 & 0.27 & -0.06 & & 0.05 & 0.16 \\
$\mathbf{5 9}$ & -1.81 & -1.58 & -0.23 & & -1.80 & -0.01 \\
\hline
\end{tabular}

*Compounds of the test set

Table 5 Statistical results for MLR and LS-SVM models

\begin{tabular}{llllll}
\hline Model & \multicolumn{2}{l}{ Train set } & & & Test set \\
\cline { 2 - 3 } \cline { 5 - 6 } & $\mathrm{R}^{2}$ & $\mathrm{SE}$ & & $\mathrm{R}^{2}$ & $\mathrm{SE}$ \\
\hline MLR & 0.702 & 0.49 & & 0.689 & 0.53 \\
LS-SVM & 0.993 & 0.10 & & 0.977 & 0.20 \\
\hline
\end{tabular}

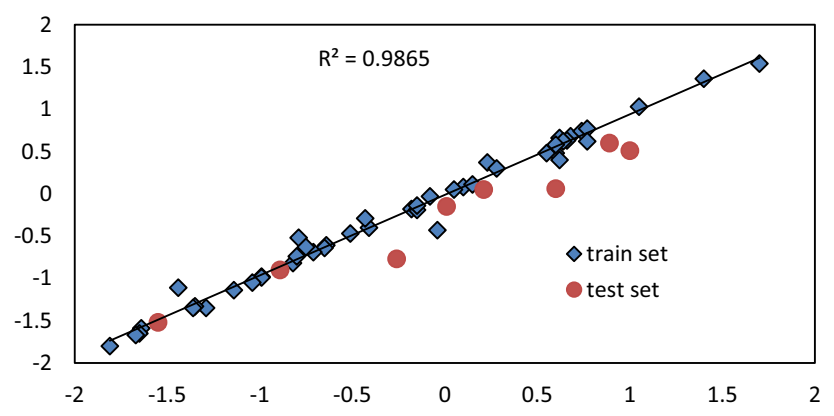

Fig. 3 The plot of LS-SVM calculated versus experimental values of $\mathrm{plC}_{50}$

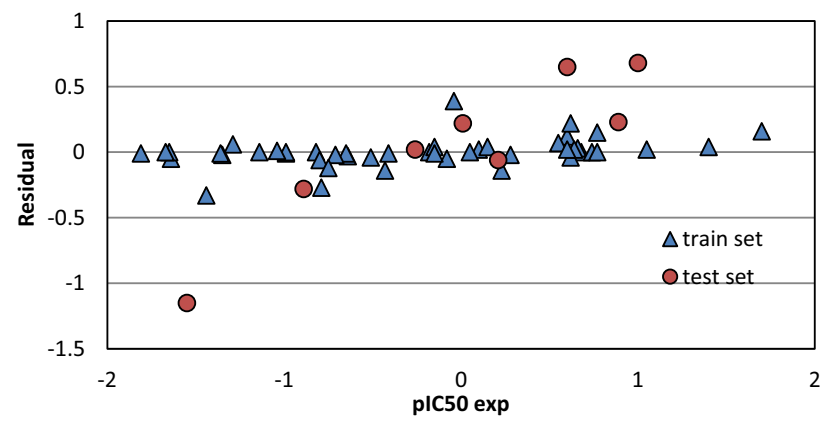

Fig. 4 The plot of MLR calculated residuals versus experimental values of $\mathrm{plC}_{50}$

calculated values of PIC50 by MLR and LS-SVM model and their corresponding residual values for training and test set were reported in Table 4. Also, statistical parameters of 2D-QSAR models were displayed in Table 5 . The better statistical values of this model are fulfilling and reveal the steadiness robustness of LS-SVM model and depict the superiority of LS-SVM over MLR model. Apart from this, the validity of the LS-SVM model was assessed using the cross-validation $\left(\mathrm{Q}^{2}\right)$ method and the obtained $\mathrm{Q}^{2}$ was 0.550 . As a result, the relationship between the selected descriptors and $\mathrm{plC}_{50}$ values should be nonlinear than linear. The plot of predicted $\mathrm{plC}_{50}$ values against experimental values was demonstrated in Fig. 3 which indicates LS-SVM well fitness with data. The residuals of predicted $\mathrm{PIC}_{50}$ values were plotted against the experimental values in Fig. 4. The scattering of residuals on each side of the zero line indicates the lack of systematic error in the developed LS-SVM model.

\subsection{D-QSAR statistical analysis}

The experimental and calculated values of PIC50 by COMFA and COMSIA model and their corresponding residual values for training and test set were reported in Table 6 . More obtained statistical parameters of established CoMFA and COMSIA model were listed in Table 7. The results of this table show the higher influence of steric field descriptors than the electrostatic ones. Figure 5 demonstrates the plot of experimental against calculated values of inhibitory activity by the CoMFA model. As shown in this figure, most points, indicating the training and test molecules, are equally dispersed along the line $y=x$ which demonstrates the good quality of CoMFA model. Also, the results of Table 7 show that the steric field has no contribution in CoMSIA modeling while electrostatic field has a larger contribution over the others. The test set statistical results imply that the CoMFA model is more reliable than CoMSIA model and can be helpful in designing novel potent CYP2A6 inhibitors.

\subsubsection{CoMFA contour maps}

To envision the information elements of the generated 3D-QSAR models, graphical maps of CoMFA model, which showed better predictability power than CoMSIA model, were created to recognize the important areas around the molecules where any alters in electrostatic and steric fields were anticipated to reduce or increase the inhibitory activity [25]. Figure 6a depicts the CoMFA steric field contour map for compound 35, more potent inhibitor in the dataset. The green colored regions of the steric field graphical plot depict an increase of inhibitory activity by bulky substituents while yellow colored regions depict a reduce of inhibitory activity by bulky substituents. As shown in this figure, the green colored contour emerging near amine group $\left(-\mathrm{NH}_{2}\right)$ indicates that bulky substituents are favorable at this area but the yellow colored contour adjacent 
Table 6 Dataset and corresponding experimental and calculated $\mathrm{pIC}_{50}$ values fo CoMFA and CoMSIA models

\begin{tabular}{|c|c|c|c|c|c|}
\hline \multirow[t]{2}{*}{ Compound } & \multirow[t]{2}{*}{$\mathrm{pIC}_{50 \exp }$} & \multicolumn{2}{|l|}{ COMFA } & \multicolumn{2}{|l|}{ COMSIA } \\
\hline & & $\mathrm{plC}_{50 \text { Pred }}$ & Residual & $\mathrm{plC}_{50 \text { Pred }}$ & Residual \\
\hline 1 & -0.64 & -0.63 & -0.01 & -0.94 & 0.30 \\
\hline 2 & -0.08 & 0.47 & -0.55 & 0.38 & -0.46 \\
\hline 3 & -0.51 & -0.42 & -0.09 & -0.84 & 0.33 \\
\hline $4^{*}$ & 1.00 & 0.51 & -0.95 & 0.45 & 0.55 \\
\hline 5 & 0.68 & 0.71 & -0.03 & 0.66 & 0.02 \\
\hline 7 & -0.18 & -0.29 & -0.11 & -0.31 & 0.13 \\
\hline 8 & 0.10 & 0.39 & -0.29 & 0.08 & 0.02 \\
\hline 9 & -0.15 & -0.74 & 0.59 & -0.19 & 0.04 \\
\hline 10 & -1.29 & -0.87 & -0.42 & -1.35 & 0.06 \\
\hline 11 & 0.66 & 0.41 & 0.25 & 0.63 & 0.03 \\
\hline $12 *$ & 0.01 & -0.21 & 0.22 & -0.15 & 0.16 \\
\hline 13 & -0.99 & -0.59 & -0.40 & -0.98 & -0.01 \\
\hline 14 & -0.65 & -0.71 & 0.06 & -0.64 & -0.01 \\
\hline $15^{*}$ & 0.60 & -0.05 & 0.65 & 0.06 & 0.54 \\
\hline 16 & -0.41 & -0.39 & -0.02 & -0.40 & -0.01 \\
\hline 17 & -0.82 & -0.89 & 0.07 & -0.82 & 0.00 \\
\hline $19 *$ & -0.89 & -0.61 & -0.28 & -0.90 & 0.01 \\
\hline 20 & -1.64 & -1.33 & -0.31 & -1.59 & -0.05 \\
\hline 21 & -0.71 & -0.91 & 0.20 & -0.69 & -0.02 \\
\hline 22 & -0.43 & -0.52 & 0.09 & -0.76 & 0.33 \\
\hline 23 & -0.99 & -0.67 & -0.32 & -0.73 & -0.26 \\
\hline $24^{*}$ & -0.26 & -0.24 & -0.02 & -0.63 & 0.37 \\
\hline 25 & -1.44 & -0.66 & -0.78 & -0.99 & -0.45 \\
\hline 26 & -0.04 & -0.41 & 0.37 & -0.27 & 0.23 \\
\hline 27 & -0.80 & -0.78 & -0.02 & -1.12 & 0.32 \\
\hline 28 & -1.65 & -1.67 & 0.02 & -1.44 & -0.21 \\
\hline 29 & 0.60 & -0.27 & 0.33 & 0.49 & 0.11 \\
\hline 30 & -0.79 & 0.08 & -0.87 & -1.00 & 0.21 \\
\hline 31 & 0.62 & 0.46 & 0.16 & 0.61 & 0.01 \\
\hline 32 & 0.62 & 0.29 & 0.33 & 0.68 & -0.06 \\
\hline 33 & 0.15 & -0.48 & 0.63 & 0.89 & -0.74 \\
\hline 34 & -1.14 & -0.92 & -0.22 & -1.28 & 0.14 \\
\hline 35 & -1.70 & 1.91 & -0.21 & 1.65 & 0.05 \\
\hline 36 & -0.08 & -0.11 & 0.03 & -0.19 & 0.11 \\
\hline 37 & 1.40 & 1.14 & 0.26 & 1.48 & -0.08 \\
\hline 38 & -0.18 & -0.33 & 0.15 & -0.46 & 0.28 \\
\hline 39 & -0.45 & 0.00 & -0.45 & -0.29 & -0.16 \\
\hline 40 & 0.74 & 0.54 & 0.20 & -0.27 & 1.10 \\
\hline 41 & 0.55 & 0.70 & -0.15 & 0.81 & -0.26 \\
\hline 42 & -1.35 & -1.24 & -0.11 & -0.87 & -0.48 \\
\hline 43 & -1.67 & -1.82 & 0.15 & -1.10 & -0.57 \\
\hline 44 & -0.75 & -0.99 & 0.19 & -0.81 & 0.06 \\
\hline $45^{*}$ & -1.55 & -1.28 & -0.27 & -1.29 & -0.26 \\
\hline 46 & 1.05 & 1.13 & -0.08 & 1.12 & -0.07 \\
\hline 47 & 0.05 & 0.04 & 0.01 & 0.17 & -0.12 \\
\hline 48 & -1.36 & -1.34 & -0.02 & -0.58 & -0.78 \\
\hline 49 & -0.15 & 0.04 & -0.19 & -0.01 & -0.14 \\
\hline 50 & -1.04 & -0.94 & -0.10 & -0.80 & -0.24 \\
\hline
\end{tabular}

Table 6 (continued)

\begin{tabular}{lcccccc}
\hline Compound & $\mathrm{pIC}_{50 \text { exp }}$ & \multicolumn{2}{l}{ COMFA } & & \multicolumn{2}{c}{ COMSIA } \\
\cline { 3 - 4 } \cline { 6 - 7 } \cline { 6 - 7 } & & $\mathrm{plC}_{50 \text { Pred }}$ & Residual & & $\mathrm{plC}_{50 \text { Pred }}$ & Residual \\
\hline $\mathbf{5 1}$ & 0.77 & 0.12 & 0.65 & & 0.77 & 0.00 \\
$\mathbf{5 2}$ & 0.23 & 0.24 & -0.01 & & -0.14 & 0.37 \\
$\mathbf{5 3 *}$ & 0.89 & 0.31 & 0.58 & & 0.29 & 0.60 \\
$\mathbf{5 4}$ & 0.28 & 0.17 & 0.11 & & -0.01 & 0.29 \\
$\mathbf{5 5}$ & 0.64 & 0.70 & -0.06 & & 0.55 & 0.09 \\
$\mathbf{5 6}$ & 0.77 & 0.32 & 0.45 & & 0.48 & 0.29 \\
$\mathbf{5 7}$ & 0.60 & 0.17 & 0.43 & & 0.39 & 0.21 \\
$\mathbf{5 8 *}$ & 0.21 & -0.14 & 0.35 & & 0.48 & -0.27 \\
$\mathbf{5 9}$ & -1.81 & -1.82 & 0.01 & & -1.63 & -0.18 \\
\hline
\end{tabular}

*Compounds of the test set

Table 7 Statistical results of CoMFA and CoMSIA model

\begin{tabular}{lll}
\hline & CoMFA & CoMSIA \\
\hline Components & & \\
$\mathrm{R}^{2}$ & 0.884 & 0.889 \\
$\mathrm{SE}$ & 0.32 & 0.31 \\
$\mathrm{R}_{\text {pred }}^{2}$ & 0.874 & 0.670 \\
$\mathrm{SE}_{\text {pred }}$ & 0.33 & 0.54 \\
$\mathrm{~F}$ values & 41.276 & 39.49 \\
$\mathrm{n}$ & 8 & 8 \\
$\mathrm{Q}^{2}$ & 0.581 & 0.554 \\
Fraction & & \\
Steric & 0.605 & 0.00 \\
Electrostatic & 0.395 & 0.505 \\
Hydrophobic & - & 0.145 \\
Hydrogen bond donor & - & 0.269 \\
Hydrogen bond acceptor & - & 0.81
\end{tabular}

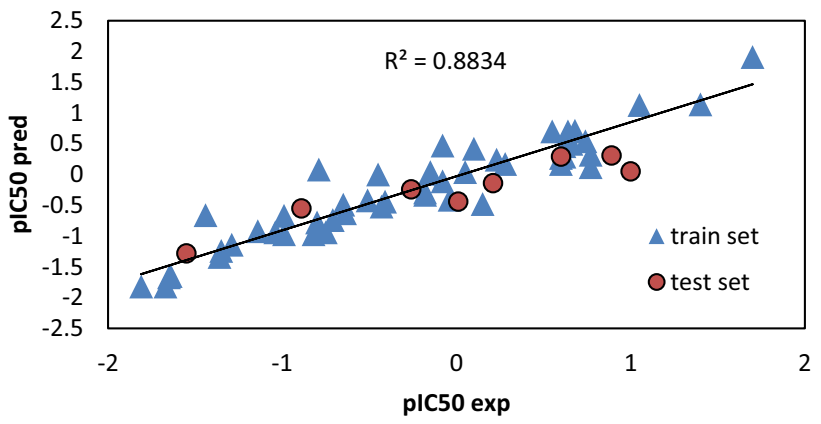

Fig. 5 The plot of CoMFA calculated versus experimental values of $\mathrm{plC}_{50}$

to this area indicates that increasing the number of bulky substituents at this area would decrease the inhibitory activity which may be due to the hindrance effect of bulky 
Fig. 6 a Steric field contour map and $\mathbf{b}$ electrostatic field contour map produced by CoMFA model
A

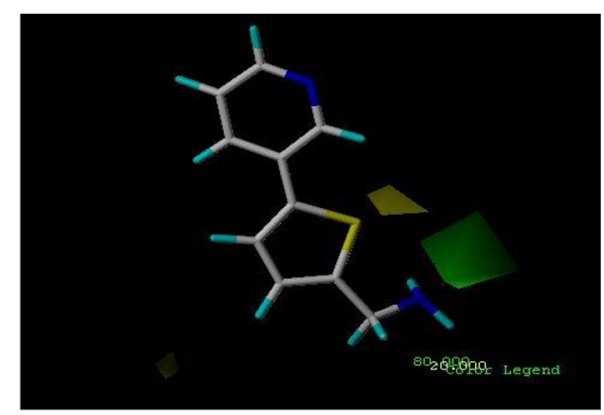

B

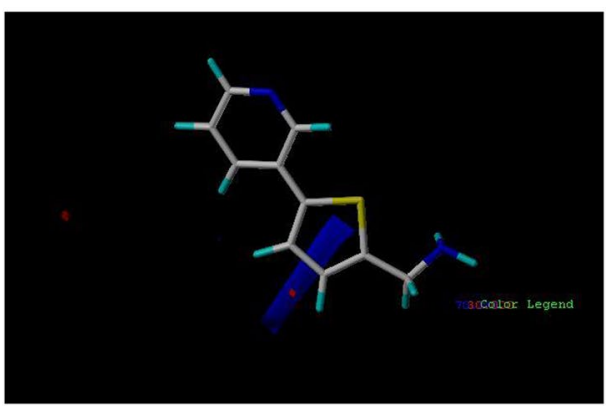

substituents on the activity of sulfur or oxygen atom at the thiophene and furan ring, respectively.

A comparison between compounds $35\left(\mathrm{plC}_{50}=1.70\right)$ and $37\left(\mathrm{plC}_{50}=1.40\right)$ shows that as the larger sulfur atom in thiophene ring replaces with a smaller oxygen atom in furan ring, inhibitory activity decreases. Also, this trend can be seen between compounds $40\left(\mathrm{plC}_{50}=0.74\right)$ and 41 $\left(\mathrm{plC}_{50}=0.55\right)$ and between compounds $42\left(\mathrm{plC}_{50}=-1.35\right)$ and $43\left(\mathrm{plC}_{50}=-1.67\right)$. The results of these comparisons reveal that as the thiophene ring attached to the amide group $\left(-\mathrm{CH}_{2}-\mathrm{NH}_{2}\right)$ replaces with furan ring, inhibitory activity decreases due to the smaller size of oxygen than sulphur atom. In contrast, increased bulky substituents to the nitrogen of amine decrease the biological activity. It may be due to the proximity of yellow colored contour to green colored contour that makes these bulky substituents unfavorable. For example, comparison between compounds $35\left(\mathrm{plC}_{50}=0.74\right)$ and $36\left(\mathrm{pIC}_{50}=-0.08\right)$ reveals that compounds 36 have an increased steric bulky substituent and as a result, its inhibitory activity dramatically drops. The same activity trends are observed among compounds $37\left(\mathrm{plC}_{50}=1.40\right)$, $38\left(\mathrm{plC}_{50}=-0.18\right)$ and $39\left(\mathrm{plC}_{50}=-0.45\right)$ and between compounds $40\left(\mathrm{plC}_{50}=0.74\right)$ and $42\left(\mathrm{plC}_{50}=-1.35\right)$ and finally between compounds $41\left(\mathrm{plC}_{50}=0.55\right)$ and 43 $\left(\mathrm{plC}_{50}=-1.67\right)$. Figure $6 \mathrm{~b}$ displays the CoMFA electrostatic field graphical plot for compound 35 , having the highest inhibitory activity in the dataset. Blue colored graphical plots depict areas where electrostatic groups are favorable whereas red colored ones depict regions where electronegative groups are favorable. As shown in this figure, the blue electrostatic contour near 3 and 4-position of thiophene ring reveals that any electropositive groups at these positions would increase the inhibitory activity. For example, the inhibitory activity of compound $4\left(\mathrm{plC}_{50}=1.00\right)$ is more than 10 -fold higher than the inhibitory activity of compound $2\left(\mathrm{plC}_{50}=-0.08\right)$, due to the electropositive $\mathrm{CH} 3$ group in the 3-position of thiophene ring.

The same activity trend can be seen between compounds $11\left(\mathrm{plC}_{50}=0.66\right)$ and $15\left(\mathrm{plC} \mathrm{C}_{50}=0.60\right)$ and also between compounds $12\left(\mathrm{plC}_{50}=0.0 .01\right)$ and 16 $\left(\mathrm{plC}_{50}=-0.41\right)$. As it can be seen from Fig. $6 \mathrm{~b}$, the red electrostatic contours are very small, which indicates that electronegative groups at 3 and 4-position of thiophene ring has a minor effect on the inhibitory activity.

Recognize the effective interactions of the new nicotine analogue on the inhibitory activity individually due to change in the number and position of the effective atom is hard and confusing. With considering such points, applying the best-fitted model by COMFA approach and draw the steric and electrostatic counter maps help to characterize the effective interactions of new nicotine analogue.

\section{Conclusion}

The main goal of the present study was to develop $2 \mathrm{D}$ and 3D-QSAR models for predicting the inhibitory activities of nicotine derivatives on CYP2A6 enzyme. Therefore, a linear MLR and a nonlinear LS-SVM methods were used to generate 2D-QSAR models. The statistical results of this investigation revealed that LS-SVM method was more reliable and had remarkable superiority over the MLR model and can be successfully employed to predict the biological activities of nicotine derivative compounds. Moreover, 3D-QSAR studies using CoMFA and CoMSIA methods were performed on the same training and test set of 2D-QSAR study. The statistical results of 3D-QSAR analyses indicated that CoMFA model was more acceptable than CoMSIA to predict the inhibitory activities of the test set. Further, the good correlation coefficient $\left(R^{2}=0.874\right)$ between observed and predicted inhibitory activity values for the test set compounds validated the predictability power of generated CoMFA model. Finally, steric and electrostatic contour maps of the CoMFA model helped to recognize the important structural features responsible for the interaction between the inhibitor molecule and receptor. Overall, the superior statistical results of the CoMFA over the CoMSIA model reveal the importance of steric and electrostatic fields in these compounds. The information extracted from this study can be helpful in the 
design of novel CYP2A6 inhibitors with enhanced inhibitory activities which may be used as a potent drug candidate.

Acknowledgements The author thanks Dr. Zahra Pahlavan Yali for academic English editing of the paper.

\section{Compliance with ethical standards}

Conflict of interest The authors declare that they have no competing interest.

\section{References}

1. Armstrong NA (2006) Pharmaceutical experimental design and interpretation. CRC Press, Boca Raton

2. Benowitz NL (1988) Pharmacologic aspects of cigarette smoking and nicotine addiction. N Engl J Med 319(20):1318-1330. https://doi.org/10.1056/NEJM198811173192005

3. Benowitz NL (2010) Nicotine addiction. N Engl J Med 362(24):2295-2303. https://doi.org/10.1056/NEJMra0809890

4. Berkman CE, Park SB, Wrighton SA, Cashman JR (1995) In vitro-in vivo correlations of human (S)-nicotine metabolism. Biochem Pharmacol 50(4):565-570. https://doi. org/10.1016/0006-2952(95)00168-y

5. Böhm M, Stürzebecher J, Klebe G (1999) Three-dimensional quantitative structure - activity relationship analyses using comparative molecular field analysis and comparative molecular similarity indices analysis to elucidate selectivity differences of inhibitors binding to trypsin, thrombin, and factor Xa. J Med Chem 42(3):458-477. https://doi.org/10.1021/jm981 062r

6. Bucholtz EC, Tropsha AE (1999) The effect of region size on CoMFA analyses. Med Chem Res 9(9):675-685

7. Burns D M (2003) Tobacco-related diseases. In: Seminars in oncology nursing. WB Saunders, vol 19, No. 4, pp 244-249. https ://doi.org/10.1053/j.soncn.2003.08.001

8. Bush BL, Nachbar RB (1993) Sample-distance partial least squares: PLS optimized for many variables, with application to CoMFA. J Comput Aid Mol Des 7(5):587-619. https://doi. org/10.1007/BF00124364

9. Chen Q, Zhao J, Fang CH, Wang D (2007) Feasibility study on identification of green, black and Oolong teas using near-infrared reflectance spectroscopy based on support vector machine (SVM). Spectrochim Acta A Mol Biomol Spectrosc 66(3):568-574. https://doi.org/10.1016/j.saa.2006.03.038

10. Clark M, Cramer RD III, Jones DM, Patterson DE, Simeroth PE (1990) Comparative molecular field analysis (CoMFA). 2. Toward its use with 3D-structural databases. Tetrahedron Comput Methodol 3(1):47-59. https://doi.org/10.1016/0898-5529(90)90120-w

11. Cortes C, Vapnik V (1995) Support-vector networks. Mach Learn 20(3):273-297. https://doi.org/10.1007/BF00994018

12. Cramer RD, Patterson DE, Bunce JD (1988) Comparative molecular field analysis (CoMFA). 1. Effect of shape on binding of steroids to carrier proteins. J Am Chem Soc 110(18):5959-5967. https://doi.org/10.1021/ja00226a005

13. Cui W, Yan X (2009) Adaptive weighted least square support vector machine regression integrated with outlier detection and its application in QSAR. Chemometr Intell Lab Syst 98(2):130-135. https://doi.org/10.1016/j.chemolab.2009.05.008

14. Davies M (1961) Multiple linear regression analysis with adjustment for class differences. J Am Stat Assoc 56(295):729-735. https://doi.org/10.1080/01621459.1961.10480657
15. Denton TT, Zhang X, Cashman JR (2005) 5-Substituted, 6-substituted, and unsubstituted 3-heteroaromatic pyridine analogues of nicotine as selective inhibitors of cytochrome P-450 2A6. J Med Chem 48(1):224-239. https://doi.org/10.1021/jm049696n

16. Devillers J, Balaban AT (eds) (2000) Topological indices and related descriptors in QSAR and QSPAR. CRC Press, Boca Raton

17. Devinyak O, Havrylyuk D, Lesyk R (2014) 3D-MoRSE descriptors explained. J Mol Graph Model 54:194-203. https://doi. org/10.1016/j.jmgm.2014.10.006

18. Ezzati $M$, Vander Hoorn S, Rodgers A, Lopez AD, Mathers $C D$, Comparative Risk Assessment Collaborating Group (2003) Estimates of global and regional potentil health gains from reducing muliple major risk factors. Lancet 362(9380):271-280. https ://doi.org/10.1016/S0140-6736(03)13968-2

19. Ferrão MF, Godoy SC, Gerbase AE, Mello C, Furtado JC, Petzhold CL, Poppi RJ (2007) Non-destructive method for determination of hydroxyl value of soybean polyol by LS-SVM using HATR/FT-IR. Anal Chim Acta 595(1-2):114-119. https://doi. org/10.1016/j.aca.2007.02.066

20. Gasteiger J, Sadowski J, Schuur J, Selzer P, Steinhauer L, Steinhauer V (1996) Chemical information in 3D space. J Chem Inf Comput Sci 36(5):1030-1037. https://doi.org/10.1021/ci960 $343+$

21. Ghose AK, Crippen GM (1986) Structure-directed quantitative structure-activity relationships I. Partition coefficients as a measure of hydrophobicity. J Comput Chem 7:565-577

22. Golbraikh A, Tropsha A (2002) Beware of q2! Journal of molecular graphics and modeling 20(4):269-276. https://doi.org/10.1016/ S1093-3263(01)00123-1

23. Hecht SS, Hoffmann D (1988) Tobacco-specific nitrosamines, an important group of carcinogens in tobacco and tobacco smoke. Carcinogenesis 9(6):875-884. https://doi.org/10.1093/ carcin/9.6.875

24. Henningfield JE, Keenan RM (1993) Nicotine delivery kinetics and abuse liability. J Consult Clin Psychol 61(5):743-750. https ://doi.org/10.1037/0022-006x.61.5.743

25. Hopfinger AJ, Tokarski JS (1997) Three-dimensional quantitative structure-activity relationship analysis. Marcel Dekker, Inc., New York, pp 105-164

26. Hukkanen J, Jacob P, Benowitz NL (2005) Metabolism and disposition kinetics of nicotine. Pharmacol Rev 57(1):79-115. https ://doi.org/10.1124/pr.57.1.3

27. HyperChem, R. (2002). 7.0 for windows, Hypercube

28. Ivanciuc O (1997) CODESSA version 2.13 for Windows. J Chem Inform Comput Sci 37(2):405-406. https://doi.org/10.1021/ci950 $193 n$

29. Karelson M (2000) Molecular descriptors in QSAR/QSPR. WileyInterscience, Hoboken

30. Klebe G, Abraham U (1993) On the prediction of binding properties of drug molecules by comparative molecular field analysis. J Med Chem 36(1):70-80. https://doi.org/10.1021/jm00053a00 9

31. Klebe G, Abraham U, Mietzner T (1994) Molecular similarity indices in a comparative analysis (CoMSIA) of drug molecules to correlate and predict their biological activity. J Med Chem 37(24):4130-4146. https://doi.org/10.1021/jm00050a010

32. Kubinyi H (1998) Comparative molecular field analysis (CoMFA). Encyclopedia Comp Chem 1:448-460. https://doi. org/10.1002/9783527618279.ch44d

33. Kuper H, Adami HO, Boffetta $P$ (2002) Tobacco use, cancer causation and public health impact. J Intern Med 251(6):455-466. https://doi.org/10.1046/j.1365-2796.2002.00993.x

34. Nakajima M, Yamagishi SI, Yamamoto H, Yamamoto T, Kuroiwa $Y$, Yokoi T (2000) Deficient cotinine formation from nicotine is attributed to the whole deletion of the CYP2A6 gene in humans. 
Clin Pharmacol Ther 67(1):57-69. https://doi.org/10.1067/ mcp.2000.103957

35. Mauri A, Consonni V, Pavan M, Todeschini R (2006) Dragon software- An easy approach to molecular descriptor calculations. Commun Math Comput Chem 56(2):237-248

36. Messina ES, Tyndale RF, Sellers EM (1997) A major role for CYP2A6 in nicotine C-oxidation by human liver microsomes. J Pharmacol Exp Ther 282(3):1608-1614

37. Peto R, Boreham J, Lopez AD, Thun M, Heath C (1992) Mortality from tobacco in developed countries: indirect estimation from national vital statistics. Lancet 339(8804):1268-1278. https://doi. org/10.1016/0140-6736(92)91600-D

38. Pianezza ML, Sellers EM, Tyndale RF (1998) Nicotine metabolism defect reduces smoking. Nature 393(6687):750. https://doi. org/10.1038/31623

39. Schuur JH, Selzer P, Gasteiger J (1996) The coding of the threedimensional structure of molecules by molecular transforms and its application to structure-spectra correlations and studies of biological activity. J Chem Inf Comput Sci 36(2):334-344. https://doi.org/10.1021/ci950164c

40. Sellers EM, Tyndale RF, Fernandes LC (2003) Decreasing smoking behaviour and risk through CYP2A6 inhibition. Drug Discov Today 8(11):487-493. https://doi.org/10.1016/S1359 -6446(03)02704-1

41. Ståhle, L., \& Wold, S. (1988). 6 multivariate data analysis and experimental design in biomedical research. In Progress in medicinal chemistry (Vol. 25, pp. 291-338). Elsevier. https://doi. org/10.1016/s0079-6468(08)70281-9

42. Stewart JJ (1989) MOPAC 6.0, Quantum chemistry program exchange. Indiana University, Bloomington
43. Suykens JA, Vandewalle J (1999) Least squares support vector machine classifiers. Neural Process Lett 9(3):293-300. https:// doi.org/10.1023/A:1018628609742

44. Sweeney CT, Fant RV, Fagerstrom KO, McGovern JF, Henningfield JE (2001) Combination nicotine replacement therapy for smoking cessation. CNS Drugs 15(6):453-467. https://doi. org/10.2165//00023210-200115060-00004

45. Todeschini R, Consonni V (2008) Handbook of molecular descriptors, vol 11. Wiley, Hoboken

46. Todeschini R, Gramatica P (1997) The WHIM theory: new 3D molecular descriptors for QSAR in environmental modelling. SAR QSAR Environ Res 7(1-4):89-115. https://doi. org/10.1080/10629369708039126

47. Vial WC (1986) Southwestern internal medicine conference: cigarette smoking and lung disease. Am J Med Sci 291(2):130-141. https://doi.org/10.1097/00000441-198602000-00011

48. Wold S, Esbensen K, Geladi P (1987) Principal component analysis. Chemometr Intell Lab Syst 2(1-3):37-52. https://doi. org/10.1016/0169-7439(87)80084-9

49. Wold S, Sjöström M, Eriksson L (2001) PLS-regression: a basic tool of chemometrics. Chemometr Intell Lab Syst 58(2):109-130. https://doi.org/10.1016/S0169-7439(01)00155-1

50. Yap CW (2011) PaDEL-descriptor- An open source software to calculate molecular descriptors and fingerprints. J Comput Chem 32(7):1466-1474. https://doi.org/10.1002/jcc.21707

Publisher's Note Springer Nature remains neutral with regard to jurisdictional claims in published maps and institutional affiliations. 